\title{
Existence of invariant measures for the stochastic damped Schrödinger equation
}

\author{
Ibrahim Ekren, Igor Kukavica, and Mohammed Ziane \\ Wednesday $19^{\text {th }}$ September, 2018
}

\author{
Departement fur Mathematik, ETH Zurich, Ramistrasse 101, CH-8092, Zurich \\ email: ibrahim.ekren@math.ethz.ch \\ Department of Mathematics, University of Southern California, Los Angeles, CA 90089 \\ e-mails: kukavica@usc.edu,ziane@usc.edu
}

\begin{abstract}
In this paper, we address the long time behavior of solutions of the stochastic Schrödinger equation in $\mathbb{R}^{d}$. We prove the existence of an invariant measure and establish asymptotic compactness of solutions, implying in particular the existence of an ergodic measure.
\end{abstract}

Mathematics Subject Classification:

Keywords: Invariant measures, stochastic Schrödinger equation, white noise, long time behavior, asymptotic compactness, tightness, Feller property, Aldous criterion

\section{Introduction}

The main purpose of the paper is to study the long time behavior of the stochastic damped Schrödinger equation

$$
d u+\left(\lambda u+i \Delta u-i|u|^{2 \sigma} u\right) d t=\Phi d W_{t}
$$

in an unbounded domain. Our main result provides the existence of an invariant measure of the Markov semigroup for the equation (1.1) driven by an additive noise. In addition, using the asymptotic compactness, we prove that the set of invariant measures is closed and convex leading to an existence of an ergodic measure.

The problem of existence of an invariant measure for stochastic partial differential equations with dissipation and in a bounded domain is now relatively well-understood with the construction of the invariant measure following the classical Krylov-Bogolyubov procedure. The smoothing properties of the equation and the boundedness of the domain guarantee the necessary compactness. For example, the existence of invariant measures for the reaction diffusion equations, for the Navier-Stokes equations, complex Ginzburg-Landau, and fractionally dissipated Euler equations was established in [CGV], F1, F2]. Also, for the primitive equations, the invariant measure was constructed in [GKVZ]. 
In the case of nondegenerate noise, a coupling method can be used to establish existence and uniqueness of the ergodic measure. For instance, in the case of Schrödinger equation with nondegenerate noise and when the domain is bounded, Debussche and Odasso established in [DO the existence of a unique ergodic measure (cf. also [DV,DZ,GMR,HM,KS,MR]).

The main goal of this paper is to address the existence of an invariant measure for the stochastic damped Schrödinger equation in an unbounded domain. The main difficulties are the the lack of smoothing and compactness properties of the solution operator in finite time. For instance, the coupling method is not expected to work in this situation since Foias-Prodi type estimates, necessary for the approach, are not available.

In order to overcome these difficulties, we establish an asymptotic compactness property of the solution operator (cf. Lemma 3.5). Namely, we prove that for every sequence of solutions resulting from $H^{1}$ bounded initial conditions and for every sequence of times diverging to $\infty$, there exists a subsequence of solutions and a sequence of times such that marginals of these solutions at these times converge in distribution in $H^{1}$. For this purpose we employ the conserved quantities used classically for the deterministic analog of the equations. We also use the energy equation approach introduced in the deterministic setting case by J. Ball $[\underline{B}$. His method was further developed to more general deterministic situations, in particular to establish the existence and regularity of attractors for the damped KdV equation [GR, R] and for the damped Schrödinger equation [G1, G2, G3, GK, GL]. Two byproducts of the asymptotic compactness property established in this paper is the existence of an invariant measure for the stochastic Schrödinger equation and the compactness of the set of invariant measures. We note that the existence and uniqueness of solutions was established by de Bouard and Debussche in DD.

The paper is organized as follows. In Section 4 we prove an abstract tightness result that links the evolution of some scalar quantities to the asymptotic compactness stated above. The main feature of the $k$-th order scalar quantity is that it is equivalent to the $H^{k}$ norm, while the drift of square of its expectation is continuous in $H^{k-1}$ norm. We also make an Aldous type continuity assumption (cf. (iii) in Definition 4.4) which allows us to use Aldous criterion [Bi] for convergence of distributions in $L_{\mathrm{loc}}^{2}$ to pass to a limiting martingale solution [D, MiR1, MiR2. We note that while the linear part is assumed to be a Schrödinger type operator $i \Delta$, our criterion can be used for more general linear operators as well after suitable adjustments. In Section 5 , we use this asymptotic compactness criterion for the Schrödinger equation by considering the first two classical Schrödinger invariants and prove the main tightness lemma. The paper is concluded by showing that the set of invariant measures is closed and convex, which implies the existence of an ergodic measure.

\section{Notations}

For functions $u, v \in L^{2}\left(\mathbb{R}^{d}\right)=L^{2}\left(\mathbb{R}^{d} ; \mathbb{C}\right)$, denote by $\|u\|_{L^{2}}$ the $L^{2}\left(\mathbb{R}^{d}\right)$ norm of $u$ and by $(u, v)=$ $\int_{\mathbb{R}^{d}} u(x) \bar{v}(x) d x$, the $L^{2}$-inner product of $u$ and $v$. We fix a basis $\left\{e_{i}\right\}_{i \geq 0}$ of $L^{2}\left(\mathbb{R}^{d}\right)$ that consists of smooth and compactly supported functions.

For a Banach space $B$ and with $T>0$ and $p \geq 1$, denote by $L^{p}([0, T] ; B)$ the space of functions from $[0, T]$ into $B$ with integrable $p$-th power over $[0, T]$ and by $C([0, T] ; B)$ the set of continuous functions 
from $[0, T]$ into $B$. Similarly to functional spaces, for $p>0$, denote by $\mathbb{L}^{p}(\Omega, B)$ the space of random variables with values in $B$ and a finite $p$-th moment.

Denote by $\Delta=\sum_{i} \partial_{i}^{2}$ the Laplace operator and by $H^{r}\left(\mathbb{R}^{d}\right)$ the Sobolev space of functions $u$ satisfying

$$
\|u\|_{H^{r}}^{2}=\int_{\mathbb{R}}(1-\Delta)^{r / 2}(u(x) \bar{u}(x)) d x<\infty,
$$

with the inner product denoted by $(u, v)_{H^{r}}$. Write $\mathcal{B}\left(H^{1}\left(\mathbb{R}^{d}\right)\right)$ for the set of Borel measurable subsets of $H^{1}\left(\mathbb{R}^{d}\right)$. Also, denote by $L_{\text {loc }}^{2}\left(\mathbb{R}^{d}\right)$ the space of locally square integrable functions which with the usual metric is a complete metric space.

For a Hilbert space $H$, we write $\operatorname{HS}\left(L^{2}, H\right)$ for the space of linear operators $\Phi: L^{2}\left(\mathbb{R}^{d}\right) \rightarrow H$ with finite Hilbert-Schmidt norm

$$
\|\Phi\|_{\mathrm{HS}\left(L^{2}, H\right)}=\left(\sum_{i=1}^{\infty}\left\|\Phi e_{i}\right\|_{H}^{2}\right)^{1 / 2} .
$$

\section{The Schrödinger equation}

We fix a probability space $(\Omega, \mathbb{F}, \mathbb{P})$ carrying a countable family of independent Brownian motions $\left\{B_{t}^{i}\right\}_{i \in \mathbb{N}, t \geq 0}$ and define the Wiener process

$$
W_{t}=\sum_{i \in \mathbb{N}} e_{i} B_{t}^{i}
$$

Fix $\lambda>0$. In this paper, we investigate the long time behavior of solutions of the stochastic damped nonlinear Schrödinger equation

$$
d u+\left(\lambda u+i \Delta u-i|u|^{2 \sigma} u\right) d t=\Phi d W_{t},
$$

on the space-time domain $[0, \infty) \times \mathbb{R}^{d}$ with an additive noise, by establishing the existence of an invariant measure and the asymptotic tightness of solutions of the equation. We emphasize that unlike in MR, Assumption H1], our problem in the whole space $\mathbb{R}^{d}$ does not allow any compact embeddings.

Recall the functionals

$$
\begin{aligned}
M(v) & =|v|_{L^{2}}^{2} \\
H(v) & =\frac{1}{2} \int_{\mathbb{R}^{d}}|\nabla v(x)|^{2} d x-\frac{1}{2 \sigma+2} \int_{\mathbb{R}^{d}}|v(x)|^{2 \sigma+2} d x
\end{aligned}
$$

which are classical invariant quantities for the Schrödinger equation. The existence of solutions for the equation (3.2) was proven in [DD. In order to be able to apply the existence results in [DD], we make the following assumptions.

Assumptions 3.1. i) $0 \leq \sigma<2 /(d-2)$ if $d \geq 3$ or $\sigma \geq 0$ if $d=1,2$.

ii) $\Phi \in H S\left(L^{2}\left(\mathbb{R}^{d}\right) ; H^{1}\left(\mathbb{R}^{d}\right)\right)$.

We now recall the existence result from [DD, Theorem 3.4, Propositions 3.2 and 3.4]. 
Theorem 3.2. Under Assumptions 3.1, for every $\mathbb{F}_{0}$ measurable, $H^{1}\left(\mathbb{R}^{n}\right)$ valued random variable $u_{0}$, there exists an $H^{1}\left(\mathbb{R}^{d}\right)$-valued and continuous solution $\left\{u_{t}\right\}_{t \geq 0}$ of (3.2) with the initial condition $u_{0}$. Additionally, the quantities $M$ and $H$ evolve as

$$
d M\left(u_{s}\right)+2 \lambda M\left(u_{s}\right) d s=2 \sum_{i} \operatorname{Re}\left(u_{s}, \Phi e_{i}\right) d B^{i}(s)+\|\Phi\|_{H S\left(L^{2} ; L^{2}\right)}^{2} d s
$$

and

$$
\begin{aligned}
& d H\left(u_{s}\right)+2 \lambda H\left(u_{s}\right) d s \\
& =\frac{\lambda \sigma}{\sigma+1} \int|u(\widetilde{s}, x)|^{2 \sigma+2} d x d \widetilde{s}-\sum_{i} \mathbb{R e}\left(\Delta u(s)+|u(s)|^{2 \sigma} u(s), \Phi e_{i}\right) d B_{s}^{i} \\
& \quad+\left(\frac{\|\nabla \Phi\|_{H S\left(L^{2} ; L^{2}\right)}^{2}}{2}-\frac{\left\|\left.u(s)\right|^{\sigma} \Phi\right\|_{H S\left(L^{2} ; L^{2}\right)}^{2}}{2}\right) d s \\
& \quad-\sigma \sum_{i}\left(|u(s)|^{2 \sigma-2},\left(\mathbb{R e}\left(\bar{u}(s) \Phi e_{i}\right)\right)^{2}\right) d s,
\end{aligned}
$$

where $|u(s)|^{\sigma} \Phi$ is the operator that to a function $v$ associates the function $|u(s)|^{\sigma} \Phi v$.

Note that the results in [DD] are given for $\lambda=0$ but one can easily pass from $\lambda=0$ to any $\lambda>0$.

\subsection{The Semigroup}

Let $u_{0} \in H^{1}\left(\mathbb{R}^{d}\right)$ be a deterministic initial condition, and let $u$ be the corresponding solution of (3.2). For all $B \in \mathcal{B}\left(H^{1}\left(\mathbb{R}^{d}\right)\right)$ we define the transition probabilities of the equation by

$$
P_{t}\left(u_{0}, B\right)=\mathbb{P}\left(u_{t} \in B\right) .
$$

For any $H^{1}\left(\mathbb{R}^{d}\right)$-valued measure $\nu$, we denote by $\left(\nu P_{t}\right)(\cdot)=\int_{H^{1}\left(\mathbb{R}^{d}\right)} P_{t}(v, \cdot) \nu(d v)$ the distribution at time $t$ of the solution of (3.2) with the initial condition having the distribution $\nu$.

For any function $\xi \in C_{b}\left(H^{1}\left(\mathbb{R}^{d}\right) ; \mathbb{R}\right)$ and $t \geq 0$, denote

$$
P_{t} \xi\left(u_{0}\right)=\mathbb{E}\left[\xi\left(u_{t}\right)\right]=\int_{H^{1}\left(\mathbb{R}^{d}\right)} \xi(v) P_{t}\left(u_{0}, d v\right) .
$$

Definition 3.3. Let $\mu$ be a probability measure on $H^{1}\left(\mathbb{R}^{d}\right)$. We say that $\mu$ is an invariant measure for $P_{t}$ if we have

$$
\int_{H^{1}\left(\mathbb{R}^{d}\right)} \xi(v) \mu(d v)=\int_{H^{1}\left(\mathbb{R}^{d}\right)} P_{t} \xi\left(v^{\prime}\right) \mu\left(d v^{\prime}\right)
$$

for all $\xi \in C_{b}\left(H^{1}\left(\mathbb{R}^{d}\right) ; \mathbb{R}\right)$ and $t \geq 0$.

\subsection{Main results concerning the Schrödinger equation}

The following statement is the main result of this paper.

Theorem 3.4. Under Assumptions 3.1, there exists an invariant measure for $P_{t}$. 
The main ingredient in the proof is the following lemma.

Lemma 3.5. Under Assumptions 3.1 the following two tightness assertions hold.

i) For all sequences of times $t_{n} \rightarrow \infty$ and $\mathbb{F}_{0}$-measurable initial conditions $u_{0}^{n} \in H^{1}\left(\mathbb{R}^{d}\right)$ with distributions $\nu^{n}$ satisfying

$$
\mathbb{E}\left[\left\|u_{0}^{n}\right\|_{H^{1}}^{4 \vee\lceil 4 d \sigma\rceil}+\left\|u_{0}^{n}\right\|_{L^{2}}^{\lceil 4 \sigma(2-d)+8\rceil]}\right] \leq R
$$

for some $R>0$, the family of measures

$$
\left\{\left(\nu^{n} P_{t_{n}}\right)(\cdot): n \in \mathbb{N}\right\}
$$

on $H^{1}\left(\mathbb{R}^{d}\right)$ is tight.

ii) For all compact sets $K \subseteq H^{1}\left(\mathbb{R}^{d}\right)$ the family of probabilities

$$
\left\{P_{s}(v, \cdot): s \in[0,1], v \in K\right\}
$$

on $H^{1}\left(\mathbb{R}^{d}\right)$ is tight.

Assuming the lemma, we now prove the main theorem. The lemma is then proven in Section 5 below. Proof of Theorem 3.4. An invariant measure is constructed using the classical Krylov-Bogolyubov theorem, which requires the Feller property of the semigroup and the tightness of averaged measures

$$
\mu_{n}(\cdot):=\frac{1}{n} \int_{0}^{n} P_{t}(0, \cdot) d t
$$

The Feller property is a consequence of [DD, Proposition 3.5]. Thus in order to conclude the proof, we only need to show tightness of the family of measures $\mu_{n}$.

Let $\epsilon>0$. Lemma 3.5 applied to the family $\left\{P_{k}(0, \cdot) ; k \in \mathbb{N}\right\}$ gives the existence of a compact set $K_{\epsilon} \subseteq H^{1}\left(\mathbb{R}^{d}\right)$ such that

$$
\sup _{k} P_{k}\left(0, K_{\epsilon}^{c}\right) \leq \frac{\epsilon}{2}
$$

We then consider the family of probabilities $\left\{P_{s}(v, \cdot): s \in[0,1], v \in K_{\epsilon}\right\}$. By the second part of Lemma 3.5, this family is tight. Therefore, there exists another compact set $A_{\epsilon} \subseteq H^{1}\left(\mathbb{R}^{d}\right)$ such that

$$
\sup _{s \in[0,1], v \in K_{\epsilon}} P_{s}\left(v, A_{\epsilon}^{c}\right) \leq \frac{\epsilon}{2} .
$$

By a direct computation

$$
\begin{aligned}
\mu_{n}\left(A_{\epsilon}^{c}\right) & =\frac{1}{n} \int_{0}^{n} P_{t}\left(0, A_{\epsilon}^{c}\right) d t=\frac{1}{n} \sum_{k=0}^{n-1} \int_{k}^{k+1} P_{t}\left(0, A_{\epsilon}^{c}\right) d t \\
& =\frac{1}{n} \sum_{k=0}^{n-1} \int_{k}^{k+1} \int_{H^{1}\left(\mathbb{R}^{d}\right)} P_{k}(0, d v) P_{t-k}\left(v, A_{\epsilon}^{c}\right) d t \\
& =\frac{1}{n} \sum_{k=0}^{n-1} \int_{k}^{k+1}\left(\int_{H^{1}\left(\mathbb{R}^{d}\right) \cap K_{\epsilon}^{c}} P_{k}(0, d v) P_{t-k}\left(v, A_{\epsilon}^{c}\right)+\int_{H^{1}\left(\mathbb{R}^{d}\right) \cap K_{\epsilon}} P_{k}(0, d v) P_{t-k}\left(v, A_{\epsilon}^{c}\right)\right) d t
\end{aligned}
$$


whence

$$
\begin{aligned}
\mu_{n}\left(A_{\epsilon}^{c}\right) & \leq \frac{1}{n} \sum_{k=0}^{n-1}\left(P_{k}\left(0, K_{\epsilon}^{c}\right)+P_{k}\left(0, K_{\epsilon}\right) \sup _{s \in[0,1], v \in K_{\epsilon}} P_{s}\left(v, A_{\epsilon}^{c}\right)\right) \\
& \leq \frac{1}{n} \sum_{k=0}^{n-1}\left(P_{k}\left(0, K_{\epsilon}^{c}\right)+\sup _{s \in[0,1], v \in K_{\epsilon}} P_{s}\left(v, A_{\epsilon}^{c}\right)\right) \leq \epsilon .
\end{aligned}
$$

We have thus shown that the set of measures $\left\{\mu_{n}\right\}$ is tight, concluding the proof of the theorem.

The rest of the paper is devoted to the proof of Lemma 3.5 and to establishing the compactness of the set of invariant measures.

\section{An abstract tightness result}

In this section, we give certain distributional convergence results that we use below to prove Lemma 3.5

Lemma 4.1. Let $k \in \mathbb{N}_{0}$, and let $\xi_{n}$ and $\xi$ be an $H^{k}\left(\mathbb{R}^{d}\right)$-valued square integrable random variables such that $\xi_{n} \rightarrow \xi$ in distribution in $L_{\text {loc }}^{2}\left(\mathbb{R}^{d}\right)$. Assume that $\mathbb{E}\left[\left\|\xi_{n}\right\|_{H^{k}}^{2}\right] \rightarrow \mathbb{E}\left[\|\xi\|_{H^{k}}^{2}\right]$ as $n \rightarrow \infty$ and suppose that the family $\left\{\left\|\xi_{n}\right\|_{H^{k}}^{2}: n \in \mathbb{N}\right\}$ is uniformly integrable. Then $\xi_{n}$ converges to $\xi$ in distribution in $H^{k}\left(\mathbb{R}^{d}\right)$.

Note that when $k=0$, we have $H^{0}\left(\mathbb{R}^{d}\right)=L^{2}\left(\mathbb{R}^{d}\right)$.

Proof Lemma 4.1. Let $\left\{f_{i}\right\}$ be a complete orthonormal system for $H^{k}\left(\mathbb{R}^{d}\right)$ consisting of smooth compactly supported functions. We first claim that

$$
\lim _{N \rightarrow \infty} \sup _{n} \mathbb{E}\left[\sum_{i=N}^{\infty}\left|\left(\xi_{n}, f_{i}\right)_{H^{k}}\right|^{2}\right]=0
$$

which then quickly implies asserted convergence. Let $\epsilon>0$. By the uniform integrability assumption, there exists $R>0$ such that

$$
\sup _{n} \mathbb{E}\left[\left\|\xi_{n}\right\|_{H^{k}}^{2} \mathbf{1}_{\left\{\left\|\xi_{n}\right\|_{H^{k}}^{2} \geq R\right\}}\right] \leq \epsilon
$$

and, by possibly enlarging $R$, we may also assume that

$$
\mathbb{E}\left[\|\xi\|_{H^{k}}^{2} \mathbf{1}_{\left\{\|\xi\|_{H^{k}}^{2} \geq R\right\}}\right] \leq \epsilon .
$$

For all $N \in \mathbb{N}$, the convergence in distribution in $L_{\text {loc }}^{2}\left(\mathbb{R}^{d}\right)$ and the fact that $\left\{f_{i}\right\}$ have compact support imply

$$
\mathbb{E}\left[\left(\sum_{i=1}^{N}\left|\left(\xi_{n}, f_{i}\right)_{H^{k}}\right|^{2}\right) \wedge R\right] \rightarrow \mathbb{E}\left[\left(\sum_{i=1}^{N}\left|\left(\xi, f_{i}\right)_{H^{k}}\right|^{2}\right) \wedge R\right]
$$

as $n \rightarrow \infty$. Since

$$
\begin{aligned}
& \left|\mathbb{E}\left[\sum_{i=1}^{N}\left|\left(\xi_{n}, f_{i}\right)_{H^{k}}\right|^{2}\right]-\mathbb{E}\left[\sum_{i=1}^{N}\left|\left(\xi, f_{i}\right)_{H^{k}}\right|^{2}\right]\right| \\
& \leq\left|\mathbb{E}\left[\left(\sum_{i=1}^{N}\left|\left(\xi_{n}, f_{i}\right)_{H^{k}}\right|^{2}\right) \wedge R\right]-\mathbb{E}\left[\left(\sum_{i=1}^{N}\left|\left(\xi, f_{i}\right)_{H^{k}}\right|^{2}\right) \wedge R\right]\right| \\
& +\mathbb{E}\left[\left\|\xi_{n}\right\|_{H^{k}}^{2} \mathbf{1}_{\left\{\left\|\xi_{n}\right\|_{H^{k}}^{2} \geq R\right\}}\right]+\mathbb{E}\left[\|\xi\|_{H^{k}}^{2} \mathbf{1}_{\left\{\|\xi\|_{H^{k}}^{2} \geq R\right\}}\right]
\end{aligned}
$$


we have that

$$
\lim _{n} \mathbb{E}\left[\sum_{i=1}^{N}\left|\left(\xi_{n}, f_{i}\right)_{H^{k}}\right|^{2}\right]=\mathbb{E}\left[\sum_{i=1}^{N}\left|\left(\xi, f_{i}\right)_{H^{k}}\right|^{2}\right] .
$$

This convergence combined with the assumption $\mathbb{E}\left[\left\|\xi_{n}\right\|_{H^{k}}^{2}\right] \rightarrow \mathbb{E}\left[\|\xi\|_{H^{k}}^{2}\right]$ implies

$$
\mathbb{E}\left[\sum_{i=N+1}^{\infty}\left|\left(\xi_{n}, f_{i}\right)_{H^{k}}\right|^{2}\right] \rightarrow \mathbb{E}\left[\sum_{i=N+1}^{\infty}\left|\left(\xi, f_{i}\right)_{H^{k}}\right|^{2}\right] .
$$

Since $\xi$ is $H^{k}\left(\mathbb{R}^{d}\right)$-square integrable, there is $N_{0} \in \mathbb{N}_{0}$ such that

$$
\mathbb{E}\left[\sum_{i=N_{0}+1}^{\infty}\left|\left(\xi, f_{i}\right)_{H^{k}}\right|^{2}\right] \leq \frac{\epsilon}{2} .
$$

Then, using (4.7), there exists $n_{\epsilon} \in \mathbb{N}$ for which

$$
\sup _{n \geq n_{\epsilon}} \mathbb{E}\left[\sum_{i=N_{0}+1}^{\infty}\left|\left(\xi_{n}, f_{i}\right)_{H^{k}}\right|^{2}\right] \leq \epsilon .
$$

The family $\left\{\left\|\xi_{n}\right\|_{H^{k}}: n=1, \ldots, n_{\epsilon}-1\right\}$ is square integrable. Therefore,

$$
\lim _{N \rightarrow \infty} \mathbb{E}\left[\sum_{i=N}^{\infty}\left|\left(\xi_{n}, f_{i}\right)_{H^{k}}\right|^{2}\right]=0, \quad n \leq n_{\epsilon}-1 .
$$

By (4.9) and (4.10), there exists $N_{1} \geq N_{0}$ such that

$$
\sup _{n \in \mathbb{N}} \mathbb{E}\left[\sum_{i=N_{1}+1}^{\infty}\left|\left(\xi_{n}, f_{i}\right)_{H^{k}}\right|^{2}\right] \leq \epsilon .
$$

Therefore, 4.1) is established.

By [Pr, Theorem 1.13], the convergence (4.1) then implies the tightness in distribution in $H^{k}\left(\mathbb{R}^{d}\right)$ of the laws of $\left\{\xi_{n}\right\}$. Note that any limiting measure can only be the distribution of $\xi$. Thus

$$
\xi_{n} \rightarrow \xi
$$

in distribution in $H^{k}\left(\mathbb{R}^{d}\right)$.

We shall work on the space $\mathcal{Z}=C\left([0, T] ; L_{\text {loc }}^{2}(\mathbb{R})\right)$. Denote by $z$ the canonical process on this space and $\mathcal{D}$ its right continuous filtration. We state our main theorem for an SPDE of the form

$$
d u(t)=(-i \Delta u(t)+b(u(t))) d t+\Phi d W_{t}
$$

with

$$
u(t) \in L_{\mathrm{loc}}^{2}(\mathbb{R})
$$

where $b: \mathbb{C} \rightarrow \mathbb{C}$ is, for simplicity, a sum of terms of the form $u^{m}|u|^{a}$ where $m \in \mathbb{N}_{0}$ and $a \geq 0$. Its maximal degree $(\max \{m+a\})$ is assumed to be less than

$$
\frac{2 d}{d-2 k}
$$

if $d>2 k$. 
Definition 4.2. A measure $\nu$ on $\mathcal{Z}$ is a martingale solution of the equation (4.13) if for all $\phi$ smooth and compactly supported functions

$$
\int_{0}^{T}\left(\left|b\left(z_{s}\right)\right|,|\phi|\right) d s<\infty, \nu \text {-a.s. }
$$

and if

$$
M_{t}^{\phi}=\left(z_{t}-z_{0}, \phi\right)-\int_{0}^{t}\left(-i \Delta z_{s}+b\left(z_{s}\right), \phi\right) d s
$$

and

$$
\left(M_{t}^{\phi}\right)^{2}-\int_{0}^{t} \sum_{i}\left(\Phi e_{i}, \phi\right)^{2} d s
$$

are $\nu$-local martingales. We say that $\nu$ is a $H^{k}$ square integrable martingale solution if

$$
\sup _{t \in[0, T]} \mathbb{E}^{\nu}\left[\left\|z_{t}\right\|_{H^{k}}^{2}\right]<\infty .
$$

Remark 4.3. i) Note that a martingale solution can be obtained from any strong solution of (4.13). Indeed, let $u$ be a solution of (4.13) on the interval $[0, T]$. Define the measure

$$
\nu(d z)=\int_{\Omega} \delta_{\left\{\left\{u_{s}(\omega)\right\}_{s \in[0, T]}\right\}}(d z) \mathbb{P}(d \omega)
$$

meaning the measure on $\mathcal{Z}$ such that for all continuous bounded $F: \mathcal{Z} \rightarrow \mathbb{R}$ we have

$$
\int_{\mathcal{Z}} F(z) \nu(d z)=\mathbb{E}\left[F\left(\{u(s)\}_{s \in[0, T]}\right)\right] .
$$

In order to facilitate the statement of the main result of this section, we introduce the concept of $H^{k}$-evolution property.

Definition 4.4. Let $k \in \mathbb{N}$. The equation (4.13) has the $H^{k}$-norm evolution property if for $i=0, \ldots, k$ there exist continuous functions $F_{i}: L_{\text {loc }}^{2} \rightarrow \mathbb{R}, \widetilde{F}_{i}: \mathbb{R} \times L_{\text {loc }}^{2} \rightarrow \mathbb{R}$, and $G_{i}: \mathbb{R} \times \mathbb{R} \times L_{\text {loc }}^{2} \rightarrow \mathbb{R}$ satisfying the following conditions.

i) For all $t, r$ the functions $F_{i}(\cdot), \widetilde{F}_{i}(t, \cdot)$ and $G_{i}(t, r, \cdot)$ are continuous in $H^{i-1}$-topology on bounded sets of $H^{i}$ (for $i=0$ we require the continuity in $L_{\text {loc }}^{2}$ on bounded sets of $L^{2}$ ).

ii) For all $t, r$ the functions $F_{i}(\cdot), \widetilde{F}_{i}(r, \cdot)$ and $G(r, \cdot)$ have at most polynomial growth in the $H^{i}$-norm.

iii) For all $H^{k}$ square integrable martingale solutions $\nu$ of (4.13), the conservation equality

$$
\mathbb{E}^{\nu}\left[\left\|z_{t}\right\|_{H^{i}}^{2}\right]-e^{-2 \lambda(t-s)} \mathbb{E}^{\nu}\left[\left\|z_{s}\right\|_{H^{i}}^{2}\right]=\mathbb{E}^{\nu}\left[F_{i}\left(z_{t}\right)-\widetilde{F}_{i}\left(t-s, z_{s}\right)\right]+\int_{s}^{t} \mathbb{E}^{\nu}\left[G_{i}\left(t-r, z_{r}\right)\right] d r
$$

holds for all $t \geq s$.

We now state a theorem, which, combined with Lemma 4.1 gives us a tightness result needed for the Krylov-Bogolyubov procedure.

Theorem 4.5. Assume that the equation (4.13) has the $H^{k}$-norm evolution property, and let $u^{n}$ be a sequence of strong solutions of (4.13) satisfying the following conditions:

a) We have a uniform bound

$$
\gamma=\sup _{r \geq 0} \sup _{k \geq i \geq 0} \sup _{n, t} \mathbb{E}\left[\left\|b\left(u_{t}^{n}\right)\right\|_{L^{1}}^{2}+\left|F_{i}\left(u_{t}^{n}\right)\right|^{2}+\left|\widetilde{F}_{i}\left(r, u_{t}^{n}\right)\right|^{2}+\left|G_{i}\left(r, u_{t}^{n}\right)\right|^{2}+\left\|u_{t}^{n}\right\|_{H^{k}}^{4}\right]<\infty .
$$


b) For every sequence of stopping times $T_{n}$ and positive numbers $\delta_{n}$ such that $\delta_{n} \rightarrow 0$ as $n \rightarrow \infty$, we have

$$
\mathbb{E}\left[\left\|u_{T_{n}+\delta_{n}}^{n}-u_{T_{n}}^{n}\right\|_{L^{2}}^{2}\right] \rightarrow 0 \text { as } n \rightarrow \infty .
$$

c) There exists a sequence $t_{n} \rightarrow \infty$ and an $H^{k}$-valued random variable $\xi$ such that $u_{t_{n}}^{n} \rightarrow \xi$ in distribution in $L_{\mathrm{loc}}^{2}$.

Then $\mathbb{E}\left[\left\|u_{t_{n}}^{n}\right\|_{H^{k}}^{2}\right] \rightarrow \mathbb{E}\left[\|\xi\|_{H^{k}}^{2}\right]$ as $n \rightarrow \infty$.

Remark 4.6. The powers in (4.21) have been chosen so we can obtain the uniform integrability of the family and then the De la Vallee Poussin's theorem can be applied.

Proof of Theorem 4.5. Since

$$
\liminf _{n} \mathbb{E}\left[\left\|u_{t_{n}}^{n}\right\|_{H^{k}}^{2}\right] \geq \mathbb{E}\left[\|\xi\|_{H^{k}}^{2}\right]
$$

we only need to prove

$$
\limsup \mathbb{E}\left[\left\|u_{t_{n}}^{n}\right\|_{H^{k}}^{2}\right] \leq \mathbb{E}\left[\|\xi\|_{H^{k}}^{2}\right] .
$$

We establish (4.24) by induction on $k$, reasoning by contradiction at each step. For $k=0$ (cf. Step 1), we use (4.22) and the Aldous's criterion to obtain a compactness of measures induced by the process $\left\{u^{n}\right\}$. Then using (4.20) for a limiting measure we obtain a contradiction.

Step 1: First we prove (4.24) for $k=0$. We assume that the convergence does not hold. This means that, passing to a subsequence, there exists $\epsilon>0$ such that

$$
\mathbb{E}\left[\left\|u_{t_{n}}^{n}\right\|_{L^{2}}^{2}\right] \geq \mathbb{E}\left[\|\xi\|_{L^{2}}^{2}\right]+\epsilon, \quad n \in \mathbb{N} .
$$

We now pick $T>0$ such that $3 \gamma^{1 / 2} e^{-2 \lambda T} \leq \epsilon$. Note that, by (4.21), the sequence $\left\{u_{t_{n}-T}^{n}\right\}$ satisfies

$$
\sup _{n} \mathbb{E}\left[\left\|u_{t_{n}-T}^{n}\right\|_{L^{2}}^{4}\right] \leq \gamma
$$

Therefore, passing to a further subsequence, there exists an $L^{2}$-valued random variable $\xi_{-T}$ such that $u_{t_{n}-T}^{n}$ converges in distribution in $L_{\mathrm{loc}}^{2}\left(\mathbb{R}^{d}\right)$ to $\xi_{-T}$. Define a sequence of measures $\nu^{n}$ on $\mathcal{Z}$ by

$$
\nu^{n}(d z)=\int_{\Omega} \delta_{\left\{\left\{u_{t_{n}-T+r}^{n}(\omega)\right\}_{r \in[0, T]}\right\}}(d z) \mathbb{P}(d \omega) .
$$

The assumption (4.22) and the Aldous criterion [B. Theorem 16.10] imply that the sequence $\left\{\nu^{n}\right\}_{n=1}^{\infty}$ is tight in distribution in $\mathcal{Z}$. Taking a further subsequence, we obtain the existence of $\nu$ such that

$$
\mathbb{E}^{\nu^{n}}[F(z)]=\mathbb{E}\left[F\left(\left\{u_{t_{n}-T+s}^{n}\right\}_{s \in[0, T]}\right)\right] \rightarrow \mathbb{E}^{\nu}[F(z)] \quad \text { as } n \rightarrow \infty, \quad F \in C_{b}(\mathcal{Z}) .
$$

Identifying the marginals, we easily see that the distribution of $z_{T}$ under $\nu$ is the same as the distribution of $\xi$. Similarly, the distribution of $z_{0}$ under $\nu$ is the same as the distribution of $\xi_{-T}$. We write the equation (4.20) at times $t_{n}$ and $t_{n}-T$ for the measure $\nu^{n}$

$$
\mathbb{E}\left[\left\|u_{t_{n}}^{n}\right\|_{L^{2}}^{2}\right]-e^{-2 \lambda T} \mathbb{E}\left[\left\|u_{t_{n}-T}^{n}\right\|_{L^{2}}^{2}\right]=\mathbb{E}\left[F_{0}\left(u_{t_{n}}^{n}\right)-\widetilde{F}_{0}\left(T, u_{t_{n}-T}^{n}\right)\right]+\int_{0}^{T} \mathbb{E}\left[G_{0}\left(T-r, u_{t_{n}-T+r}^{n}\right)\right] d r .
$$


We claim that by the assumptions (i), (ii), and (a), we have sufficient integrability and continuity at the right hand side of the equation to use the convergence of $\nu^{n}$ to $\nu$ and pass to the limit to obtain

$$
\lim _{n}\left(\mathbb{E}\left[\left\|u_{t_{n}}^{n}\right\|_{L^{2}}^{2}\right]-e^{-2 \lambda T} \mathbb{E}\left[\left\|u_{t_{n}-T}^{n}\right\|_{L^{2}}^{2}\right]\right)=\mathbb{E}^{\nu}\left[F_{0}\left(z_{T}\right)-\widetilde{F}_{0}\left(T, z_{0}\right)\right]+\int_{0}^{T} \mathbb{E}^{\nu}\left[G_{0}\left(T-r, z_{r}\right)\right] d r
$$

Indeed, the convergence of $\nu^{n}$ to $\nu$ in $\mathcal{Z}$ implies that for all $s \in[0, T]$ and every function $\xi: L_{\text {loc }}^{2}\left(\mathbb{R}^{d}\right) \rightarrow \mathbb{R}$ continuous and bounded we have

$$
\mathbb{E}\left[\xi\left(u_{t_{n}-T+s}^{n}\right)\right] \rightarrow \mathbb{E}^{\nu}\left[\xi\left(z_{s}\right)\right]
$$

Note that, by the assumption (i), the mappings $F_{0}(\cdot), \widetilde{F}_{0}(T, \cdot)$, and $G_{0}(T-s, \cdot)$ are continuous in $L_{\text {loc }}^{2}\left(\mathbb{R}^{d}\right)$ on bounded sets of $L^{2}\left(\mathbb{R}^{d}\right)$. Additionally the assumption (ii) and the uniform bound (4.21) allows us to truncate $F_{0}(\cdot), \widetilde{F}_{0}(T, \cdot)$, and $G_{0}(T-s, \cdot)$ when they are large in order to obtain

$$
\mathbb{E}\left[\psi\left(u_{t_{n}-T+s}^{n}\right)\right] \rightarrow \mathbb{E}^{\nu}\left[\psi\left(z_{s}\right)\right]
$$

for $\psi=F_{0}(\cdot), \psi=\widetilde{F}(T, \cdot)$, and $\psi=G_{0}(T-s, \cdot)$. Thus

$$
\lim _{n}\left(\mathbb{E}\left[\left\|u_{t_{n}}^{n}\right\|_{L^{2}}^{2}\right]-e^{-2 \lambda T} \mathbb{E}\left[\left\|u_{t_{n}-T}^{n}\right\|_{L^{2}}^{2}\right]\right)=\mathbb{E}^{\nu}\left[F_{0}\left(z_{T}\right)-\widetilde{F}_{0}\left(T, z_{0}\right)\right]+\int_{0}^{T} \mathbb{E}^{\nu}\left[G_{0}\left(T-r, z_{r}\right)\right] d r .
$$

We shall show in Step 3 that $\nu$ is a $L^{2}$-square integrable martingale solution of (4.13). Using this result and by the assumption (4.20) one has

$$
\mathbb{E}^{\nu}\left[F_{0}\left(z_{T}\right)-\widetilde{F}_{0}\left(T, z_{0}\right)\right]+\int_{0}^{T} \mathbb{E}^{\nu}\left[G_{0}\left(T-r, z_{r}\right)\right] d r=\mathbb{E}^{\nu}\left[\left\|z_{T}\right\|_{L^{2}}^{2}\right]-e^{-2 \lambda T} \mathbb{E}^{\nu}\left[\left\|z_{0}\right\|_{L^{2}}^{2}\right] .
$$

Noting the bound (4.21), we may pass to the limit and obtain

$$
\begin{aligned}
\lim _{n}( & \left.\mathbb{E}\left[\left\|u_{t_{n}}^{n}\right\|_{L^{2}}^{2}\right]-e^{-2 \lambda T} \mathbb{E}\left[\left\|u_{t_{n}-T}^{n}\right\|_{L^{2}}^{2}\right]\right) \\
& =\mathbb{E}^{\nu}\left[\left\|z_{T}\right\|_{L^{2}}^{2}\right]-e^{-2 \lambda T} \mathbb{E}^{\nu}\left[\left\|z_{0}\right\|_{L^{2}}^{2}\right] \\
& =\mathbb{E}\left[\|\xi\|_{L^{2}}^{2}\right]-e^{-2 \lambda T} \mathbb{E}\left[\|\xi-T\|_{L^{2}}^{2}\right]
\end{aligned}
$$

By convergence of $u_{t_{n}-T}^{n}$ to $\xi_{-T}$ in distribution in $L_{\text {loc }}^{2}$ and the Fatou's lemma, we obtain

$$
\mathbb{E}\left[\left\|\xi_{-T}\right\|_{L^{2}}^{2}\right] \leq \liminf _{n} \mathbb{E}\left[\left\|u_{t_{n}-T}^{n}\right\|_{L^{2}}^{2}\right] \leq \gamma^{1 / 2} .
$$

Using (4.25) and (4.35), we obtain

$$
\begin{aligned}
\epsilon & \leq \liminf _{n} \mathbb{E}\left[\left\|u_{t_{n}}^{n}\right\|_{L^{2}}^{2}\right]-\mathbb{E}\left[\|\xi\|_{L^{2}}^{2}\right] \\
& \leq \limsup _{n} e^{-2 \lambda T}\left(\mathbb{E}\left[\left\|u_{t_{n}-T}^{n}\right\|_{L^{2}}^{2}\right]-\mathbb{E}\left[\left\|\xi_{-T}\right\|_{L^{2}}^{2}\right]\right) \leq \gamma^{1 / 2} e^{-2 \lambda T} \leq \frac{2 \epsilon}{3}
\end{aligned}
$$

which is a contradiction.

Step 2: Now we prove that $\nu$ is a $L^{2}$-square integrable martingale solution of (4.13). Note that the uniform bound (4.21), the lower semicontinuity of the $L^{2}$ norm with respect to the $L_{\text {loc }}^{2}$ topology, and the distributional convergence of $\nu^{n}$ to $\nu$ give that for all $t \in[0, T]$

$$
\mathbb{E}^{\nu}\left[\left\|z_{t}\right\|_{L^{2}}^{2}\right] \leq \gamma
$$


Additionally the choice of the power for $b$ implies that the mapping $z \in \mathcal{Z} \rightarrow M_{t}^{\phi}(z)$ is continuous. Thus for all $f$ bounded continuous

$$
\lim _{n} \mathbb{E}^{\nu^{n}}\left[f\left(M_{t}^{\phi}\right)\right]=\mathbb{E}^{\nu}\left[f\left(M_{t}^{\phi}\right)\right] .
$$

For all $\phi$ smooth, there exists $K_{\phi, T}$ depending only on $\phi$ and $T$ such that

$$
\left|M_{t}^{\phi}\right|^{2} \leq K_{\phi, T}\left(\left\|z_{0}\right\|_{L^{2}}^{2}+\left\|z_{t}\right\|_{L^{2}}^{2}+\int_{0}^{t}\left\|z_{r}\right\|_{L^{2}}^{2}+\left\|b\left(z_{r}\right)\right\|_{L^{1}}^{2} d r\right), \quad t \in[0, T] .
$$

We will use these points to prove that $M_{t}^{\phi}$ is a martingale under $\nu$. We fix a family of smooth truncation functions $\Psi_{R}$ satisfying $\left|\Psi_{R}\right| \leq 2 R$ and $\Psi_{R}(x)=x$ if $|x| \leq R$. For $0 \leq s_{1} \leq s_{2} \leq \ldots \leq s_{m} \leq$ $s \leq t \leq T$ smooth compactly supported functions functions $\phi_{i}$ and a random variable of the form $F=F\left(\int \phi_{1} z_{s_{1}} d x, \ldots, \int \phi_{m} z_{s_{m}} d x\right)$ smooth and bounded by 1 , we have the equalities

$$
\begin{aligned}
\left|\mathbb{E}^{\nu}\left[\left(M_{t}^{\phi}-M_{s}^{\phi}\right) F\right]\right| & =\left|\mathbb{E}^{\nu}\left[\left(\Psi_{R}\left(M_{t}^{\phi}\right)-\Psi_{R}\left(M_{s}^{\phi}\right)\right) F\right]\right|+\mathbb{E}^{\nu}\left[\left|\Psi_{R}\left(M_{t}^{\phi}\right)-M_{t}^{\phi}\right|\right]+\mathbb{E}^{\nu}\left[\left|\Psi_{R}\left(M_{s}^{\phi}\right)-M_{s}^{\phi}\right|\right] \\
& =\lim _{n}\left|\mathbb{E}^{\nu^{n}}\left[\left(\Psi_{R}\left(M_{t}^{\phi}\right)-\Psi_{R}\left(M_{s}^{\phi}\right)\right) F\right]\right|+\frac{1}{R}\left(\mathbb{E}^{\nu}\left[\left|M_{t}^{\phi}\right|^{2}\right]+\mathbb{E}^{\nu}\left[\left|M_{s}^{\phi}\right|^{2}\right]\right) \\
& =\lim _{n}\left|\mathbb{E}^{\nu^{n}}\left[\left(M_{t}^{\phi}-M_{s}^{\phi}\right) F\right]\right|+\frac{4 T \gamma K_{\phi, T}}{R} .
\end{aligned}
$$

Note that by the martingale property of $M_{t}^{\phi}$ under $\nu^{n}$ we have $\mathbb{E}^{\nu^{n}}\left[\left(M_{t}^{\phi}-M_{s}^{\phi}\right) F\right]=0$. Thus, taking $R$ to infinity we get $\mathbb{E}^{\nu}\left[\left(M_{t}^{\phi}-M_{s}^{\phi}\right) F\right]=0$ which is sufficient to claim that $M_{t}^{\phi}$ is a $\nu$ martingale. Due to the smoothness of $\phi$, the continuity of $z$ in $L_{\mathrm{loc}}^{2}$ and (4.38), $M_{t}^{\phi}$ is a continuous and square integrable martingale under $\nu$. We now proceed to characterize its quadratic variation.

By the definition of martingale solutions under $\nu^{n}$ the process $M_{t}^{\phi} / \sqrt{\sum_{i}\left(\Phi e_{i}, \phi\right)^{2}}$ is a Brownian motion and thus has Gaussian independent increments. By the distributional convergence of $\nu^{n}$ and the continuity of $M_{t}^{\phi} / \sqrt{\sum_{i}\left(\Phi e_{i}, \phi\right)^{2}}$ in the $L_{\text {loc }}^{2}$ topology with respect to $z$ the distribution and the independence of the increments still hold under $\nu$. Thus the continuous process $M_{t}^{\phi} / \sqrt{\sum_{i}\left(\Phi e_{i}, \phi\right)^{2}}$ is a Brownian motion under $\nu$ which implies that (4.16) holds under $\nu$.

Step 3: For the induction step, assume that $\mathbb{E}\left[\left\|u_{t_{n}}^{n}\right\|_{H^{r}}^{2}\right] \rightarrow \mathbb{E}\left[\|\xi\|_{H^{r}}^{2}\right]$ as $n \rightarrow \infty$ for $r=0, \ldots, k-1$. We need to show that

$$
\mathbb{E}\left[\left\|u_{t_{n}}^{n}\right\|_{H^{k}}^{2}\right] \rightarrow \mathbb{E}\left[\|\xi\|_{H^{k}}^{2}\right] .
$$

Note that using Lemma 4.1 at each step of the induction one can also show that

$$
u_{t_{n}}^{n} \rightarrow \xi
$$

in distribution in $H^{r}\left(\mathbb{R}^{d}\right)$ for all $r \leq k-1$.

In order to obtain a contradiction, assume that the convergence we are proving does not hold. This means that, up to a subsequence, there exists $\epsilon>0$ such that

$$
\mathbb{E}\left[\left\|u_{t_{n}}^{n}\right\|_{H^{k}}^{2}\right] \geq \mathbb{E}\left[\|\xi\|_{H^{k}}^{2}\right]+\epsilon, \quad n \in \mathbb{N} .
$$

Similarly to the previous step, we introduce $T$ such that $3 \gamma^{1 / 2} e^{-2 \lambda T} \leq \epsilon$ and define the measures $\nu^{n}$ on $\mathcal{Z}$. We also prove similarly that there exist an $H^{k}$-valued random variable $\xi_{-T}$, a distribution $\nu$ on $\mathcal{Z}$ 
which is a $H^{k}$ square integrable solution of (4.13) and a subsequence of $t_{n}$ (still denoted $t_{n}$ ) such that $\nu^{n} \rightarrow \nu$ on $\mathcal{Z}$ as $n \rightarrow \infty$ and $u_{t_{n}-T}^{n} \rightarrow \xi_{-T}$ in distribution in $L_{\mathrm{loc}}^{2}\left(\mathbb{R}^{d}\right)$. Note that for all $s \in[0, T]$ the family $u_{t_{n}-T+s}^{n}$ converges in distribution in $L_{\text {loc }}^{2}\left(\mathbb{R}^{d}\right)$ to the distribution of $z_{s}$ under $\nu$. Therefore, using the induction hypothesis on the family $u_{t_{n}-T+s}^{n}$ and $z_{s}$ we obtain

$$
u_{t_{n}-T+s}^{n} \rightarrow z_{s}
$$

in distribution in $H^{r}\left(\mathbb{R}^{d}\right)$ and

$$
\mathbb{E}\left[\left\|u_{t_{n}-T+s}^{n}\right\|_{H^{r}}^{2}\right] \rightarrow \mathbb{E}^{\nu}\left[\left\|z_{s}\right\|_{H^{r}}^{2}\right]
$$

for $r \leq k-1$ as $n \rightarrow \infty$.

We first use (4.20) on $\nu^{n}$ for $k$ to obtain

$$
\mathbb{E}\left[\left\|u_{t_{n}}^{n}\right\|_{H^{k}}^{2}\right]-e^{-2 \lambda T} \mathbb{E}\left[\left\|u_{t_{n}-T}^{n}\right\|_{H^{k}}^{2}\right]=\mathbb{E}\left[F_{k}\left(u_{t_{n}}^{n}\right)-\widetilde{F}_{k}\left(T, u_{t_{n}}^{n}\right)\right]+\int_{0}^{T} \mathbb{E}\left[G_{k}\left(T-s, u_{t_{n}-T+s}^{n}\right)\right] d s .
$$

We have proven that the distribution of $u_{t_{n}-T+s}^{n}$ converges in distribution in $H^{k-1}\left(\mathbb{R}^{d}\right)$ to the distribution of $z_{s}$ under $\nu$. Similarly to the previous step, we have enough integrability and continuity on the right hand side of the equation to use this convergence and pass to the limit to obtain

$$
\lim _{n} \mathbb{E}\left[\left\|u_{t_{n}}^{n}\right\|_{H^{k}}^{2}\right]-e^{-2 \lambda T} \mathbb{E}\left[\left\|u_{t_{n}-T}^{n}\right\|_{H^{k}}^{2}\right]=\mathbb{E}^{\nu}\left[F_{k}\left(z_{T}\right)-\widetilde{F}_{k}\left(T, z_{0}\right)\right]+\int_{0}^{T} \mathbb{E}^{\nu}\left[G_{k}\left(T-s, z_{s}\right)\right] d s .
$$

Using Fatou's lemma and (4.21) we have that $\nu$ is a $H^{k}\left(\mathbb{R}^{d}\right)$ square integrable solution of (4.13). Thus, by assumption, Definition 4.4 (iii) gives

$$
\mathbb{E}^{\nu}\left[\left\|z_{T}\right\|_{H^{k}}^{2}\right]-e^{-2 \lambda T} \mathbb{E}^{\nu}\left[\left\|z_{0}\right\|_{H^{k}}^{2}\right]=\mathbb{E}^{\nu}\left[F_{k}\left(z_{T}\right)-\widetilde{F}_{k}\left(T, z_{0}\right)\right]+\int_{0}^{T} \mathbb{E}^{\nu}\left[G_{k}\left(T-s, z_{s}\right)\right] d s,
$$

which implies

$$
\lim _{n}\left(\mathbb{E}\left[\left\|u_{t_{n}}^{n}\right\|_{H^{k}}^{2}\right]-e^{-2 \lambda T} \mathbb{E}\left[\left\|u_{t_{n}-T}^{n}\right\|_{H^{k}}^{2}\right]\right)=\mathbb{E}^{\nu}\left[\left\|z_{T}\right\|_{H^{k}}^{2}\right]-e^{-2 \lambda T} \mathbb{E}^{\nu}\left[\left\|z_{0}\right\|_{H^{k}}^{2}\right] .
$$

Using the same arguments as in the previous step, we obtain a contradiction.

\section{Proofs of tightness for the Schrödinger equation}

We now return to the Schrödinger equation (3.2). We fix $\lambda>0$; thus all the constants are allowed to depend on $\lambda$. Also, recall that we impose Assumptions 3.1 on $\sigma$ and $\Phi$.

Lemma 5.1. For every $k \in \mathbb{N}$ we have

$$
\sup _{t \geq 0} \mathbb{E}\left[M(u(s))^{k}\right] \leq C_{k}\left(\mathbb{E}\left[\left|M\left(u_{0}\right)\right|^{k}\right]+1\right)
$$

and

$$
\sup _{t \geq 0} \mathbb{E}\left[H(u(s))^{k}\right] \leq C_{k}\left(\mathbb{E}\left[\left|H\left(u_{0}\right)\right|^{k}\right]+1\right)
$$

where $C_{k} \geq 0$ is a constant. 
Proof of Lemma 5.1, Using similar ideas as in [EKZ], one can show that the local martingale appearing in (3.5) is a martingale. Thus we have

$$
\mathbb{E}[M(u(t))]+2 \lambda \int_{0}^{t} \mathbb{E}[M(u(s))] d s=\mathbb{E}\left[M\left(u_{0}\right)\right]+t\|\Phi\|_{H S\left(L^{2} ; L\right)}^{2} .
$$

Solving this ODE for $\mathbb{E}[M(u(t))]$, we get

$$
\mathbb{E}[M(u(t))]=e^{-2 \lambda t} \mathbb{E}\left[M\left(u_{0}\right)\right]+\|\Phi\|_{H S\left(L^{2} ; L^{2}\right)}^{2} \int_{0}^{t} e^{-2 \lambda(t-s)} d s \leq \mathbb{E}\left[M\left(u_{0}\right)\right]+\frac{1}{2 \lambda}\|\Phi\|_{H S\left(L^{2} ; L^{2}\right)}^{2}
$$

which proves (5.1) for $k=1$. For general $k$ we proceed by induction. We assume the existence of $C_{k}$ for a given $k \geq 1$ and apply Ito's lemma to $M(u(t))^{k+1}$ to obtain

$$
\begin{aligned}
& d M^{k+1}(u(t))+2(k+1) \lambda M^{k+1}(u(t)) d t \\
& =(k+1) M^{k}(u(t))\|\Phi\|_{H S\left(L^{2} ; L^{2}\right)}^{2} d t \\
& \quad+\frac{k(k+1)}{2} M^{k-1}(u(t)) \sum_{i} \operatorname{Re}\left(u(t), \Phi e_{i}\right)^{2} d t+\widetilde{M}
\end{aligned}
$$

where similarly $\widetilde{M}$ can be shown to be a martingale. Thus the function $\mathbb{E}\left[M^{k+1}(u(t))\right]$ satisfies the ODE

$$
\begin{aligned}
(\mathbb{E}[ & \left.\left.M^{k+1}(u(t))\right]\right)^{\prime}+2(k+1) \lambda \mathbb{E}\left[M^{k+1}(u(t))\right] \\
& =(k+1) \mathbb{E}\left[M^{k}(u(t))\|\Phi\|_{H S\left(L^{2} ; L^{2}\right)}^{2}\right]+\frac{k(k+1)}{2} \mathbb{E}\left[M^{k-1}(u(t)) \sum_{i} \operatorname{Re}\left(u(t), \Phi e_{i}\right)^{2}\right]=: g_{k}(t),
\end{aligned}
$$

where $g_{k}$ is a bounded function of $t$ by the induction assumption. By solving this ODE, we see that the function $\mathbb{E}\left[M^{k+1}(u(t))\right]$ is bounded. Repeating the same argument for $H^{k}(u(t))$ we obtain (5.2).

In order to obtain the tightness of the averaged measures, we use Lemma 4.1 and Theorem 4.5 The last ingredient we need is the following lemma.

Lemma 5.2. Under the assumptions of Lemma 3.5, for all stopping times $T_{n}$ and real numbers $\delta_{n}$ such that $\delta_{n} \rightarrow 0$ as $n \rightarrow \infty$, we have

$$
\mathbb{E}\left[\left\|u_{T_{n}+\delta_{n}}^{n}-u_{T_{n}}^{n}\right\|_{L^{2}}^{2}\right] \rightarrow 0 \text { as } n \rightarrow \infty
$$

Proof of Lemma 5.2. We denote by $S^{\lambda}$ the semigroup associated with the linear part of the equation. With this notation, we have

$$
\begin{gathered}
u_{T_{n}+\delta_{n}}^{n}-u_{T_{n}}^{n}=S^{\lambda}\left(\delta_{n}\right) u_{T_{n}}^{n}-u_{T_{n}}+i \int_{0}^{\delta_{n}} S^{\lambda}\left(\delta_{n}-s\right)\left(\left|u^{n}\left(T_{n}+s\right)\right|^{2 \sigma} u^{n}(s)\right) d s \\
+\int_{0}^{\delta_{n}} S^{\lambda}\left(\delta_{n}-s\right) \Phi d W_{T_{n}+s} .
\end{gathered}
$$


The lemma would follow from the following three convergence statements:

$$
\begin{aligned}
& \mathbb{E}\left[\left\|S^{\lambda}\left(\delta_{n}\right) u_{T_{n}}^{n}-u_{T_{n}}^{n}\right\|_{L^{2}}^{2}\right] \rightarrow 0, \\
& \mathbb{E}\left[\left\|\int_{0}^{\delta_{n}} S^{\lambda}\left(\delta_{n}-s\right)\left(\left|u^{n}\left(T_{n}+s\right)\right|^{2 \sigma} u^{n}(s)\right) d s\right\|_{L^{2}}^{2}\right] \rightarrow 0, \\
& \mathbb{E}\left[\left\|\int_{0}^{\delta_{n}} S^{\lambda}\left(\delta_{n}-s\right) \Phi d W_{T_{n}+s}\right\|_{L^{2}}^{2}\right] \rightarrow 0 .
\end{aligned}
$$

By PDE arguments, the first convergence is obvious. For the second convergence (5.10), we simply write

$$
\begin{aligned}
& \mathbb{E}\left[\left\|\int_{0}^{\delta_{n}} S^{\lambda}\left(\delta_{n}-s\right)\left(\left|u\left(T_{n}+s\right)\right|^{2 \sigma} u(s)\right) d s\right\|_{L^{2}}^{2}\right] \\
& \quad \leq \delta_{n} \int_{0}^{\delta_{n}} \mathbb{E}\left[\left\|S^{\lambda}\left(\delta_{n}-s\right)\left(\left|u\left(T_{n}+s\right)\right|^{2 \sigma} u(s)\right)\right\|_{L^{2}}^{2}\right] d s .
\end{aligned}
$$

Given the uniform bounds (5.1), the integrand is uniformly bounded and the convergence thus holds. For the third convergence (5.11), we use the the Burkholder-Davis-Gundy Inequality [DZ, Lemma 5.24] and obtain

$$
\mathbb{E}\left[\left\|\int_{0}^{\delta_{n}} S^{\lambda}\left(\delta_{n}-s\right) \Phi d W_{T_{n}+s}\right\|_{L^{2}}^{2}\right] \leq \int_{0}^{\delta_{n}}\left\|S^{\lambda}\left(\delta_{n}-s\right) \Phi\right\|_{H S\left(L^{2}, L^{2}\right)}^{2} d s \rightarrow 0
$$

as $n \rightarrow \infty$.

\subsection{Proof of Lemma 3.5}

Proof of (i): We show that the assumptions of Theorem 4.5 with $k=1$ are satisfied for the equation (3.2) and the set $\left\{\left(\nu^{n} P_{t_{n}}\right)(\cdot): n \in \mathbb{N}\right\}$ is relatively weakly compact over $H^{1}\left(\mathbb{R}^{d}\right)$. We define

$$
\begin{aligned}
& F_{0}=\widetilde{F}_{0}=0 \\
& G_{0}(t, r, v):=e^{-2 \lambda(t-r)}\|\Phi\|_{H S\left(L^{2}, L^{2}\right)}^{2}, \\
& F_{1}(v)=\frac{1}{2 \sigma+2} \int|v(x)|^{2 \sigma+2} d x \\
& \widetilde{F}_{1}(r, v):=e^{-2 \lambda r} \frac{1}{2 \sigma+2} \int|v(x)|^{2 \sigma+2} d x \\
& G_{1}(r, v):=e^{-2 \lambda r}\left(\int_{\mathbb{R}^{d}}|v(x)|^{2 \sigma+2} d x+\|\nabla \Phi\|_{H S\left(L^{2}, L^{2}\right)}^{2}\right. \\
& \left.\quad-\left\|\left.v\right|^{\sigma} \Phi\right\|_{H S\left(L^{2}, L^{2}\right)}^{2}-\sigma \sum_{i} \operatorname{Re}\left(|v|^{2 \sigma-2} v^{2},\left(\Phi e_{i}\right)^{2}\right)\right) .
\end{aligned}
$$

We apply the Gagliardo-Nirenberg interpolation inequality to obtain

$$
\|v\|_{L^{2 \sigma+2}}^{2 \sigma+2} \leq C\|v\|_{H^{1}}^{d \sigma}\|v\|_{L^{2}}^{\sigma(2-d)+2}
$$

which shows that $F_{1}(\cdot), \widetilde{F}_{1}(r, \cdot)$, and $G_{1}(r, \cdot)$ are continuous in $L^{2}\left(\mathbb{R}^{d}\right)$ on bounded sets of $H^{1}\left(\mathbb{R}^{d}\right)$. They also have at most polynomial growth in $H^{1}\left(\mathbb{R}^{d}\right)$ and given the bounds on $u_{0}^{n}$ and Lemma (5.1), with 
$b(u)=|u|^{2 \sigma} u$, we have the bound (4.21). Additionally, given the assumption 3.1 on $\sigma$, we can easily verify that the degree of $b$ satisfy for $d \geq 2$,

$$
2 \sigma+1<\frac{d+2}{d-2} \leq \frac{2 d}{d-2} .
$$

Since $\nu$ is a $H^{1}$-square integrable martingale solution of (3.2), by [D, Theorem 2.4], we can extend the probability space $(\mathcal{Z}, \mathcal{D}, \nu)$ to obtain a family of Brownian motions $\hat{B}^{i}$ such that the $H^{-1}\left(\mathbb{R}^{d}\right)$-valued continuous martingale $M_{t}=z(t)-z(0)+\int_{0}^{t}\left(\lambda z(s)-i \Delta z(s)-i|z(s)|^{2 \sigma} z(s)\right) d s$ can be represented as

$$
d M_{t}=\sum_{i} \Phi e_{i} d \hat{B}_{t}^{i}
$$

Similarly to [DD, Propositions 3.2 and 3.3], we apply Ito's lemma to $M(z(t))$ and $H(z(t))$ on this probability space to obtain that (4.20) holds for $i=0,1$ under $\nu$. This shows that the equation (3.2) has the $H^{1}$-norm evolution property.

Next, we consider the sequence of measures $\left(\nu^{n} P_{t_{n}}\right)(d v)$ as measures on the space $L_{\mathrm{loc}}^{2}\left(\mathbb{R}^{d}\right)$. Denote by $B_{k} \subseteq \mathbb{R}^{d}$ the ball with radius $k$, centered at the origin. Given the uniform estimates (5.2), by the compact embedding of the space $H^{1}\left(B_{k}\right)$ in $L^{2}\left(B_{k}\right)$ and a successive application of Prokhorov's theorem, we obtain that there exists a subsequence of $\left\{t_{n}, u_{0}^{n}\right\}$, which we still denote $\left\{t_{n}, u_{0}^{n}\right\}$, and a distribution $\mu$ on $H^{1}\left(\mathbb{R}^{d}\right)$ such that

$$
\left(\nu^{n} P_{t_{n}}\right)(d v) \rightarrow \mu \text { in distribution in } L_{\text {loc }}^{2}\left(\mathbb{R}^{d}\right) .
$$

We also note that the solutions $u^{n}$ satisfy the assumptions (iv) and (v) as consequences of (5.2) and (5.7) respectively. Thus by Lemma 4.1 and Theorem 4.5, the convergence

$$
\left(\nu^{n} P_{t_{n}}\right)(d v) \rightarrow \mu
$$

is in fact in distribution in $H^{1}\left(\mathbb{R}^{d}\right)$ which is what we claimed.

Proof of (ii): We choose $\left(s_{n}, v_{n}\right) \in[0, T] \times K$. By the compactness of the two sets there exist a subsequence of $\left(s_{n}, v_{n}\right)$, still denoted $\left(s_{n}, v_{n}\right)$, and $(s, v) \in[0,1] \times K$ such that $\left(s_{n}, v_{n}\right) \rightarrow(s, v)$. We claim that $P_{s_{n}}\left(v_{n}, \cdot\right)$ converges in distribution in $H^{1}\left(\mathbb{R}^{d}\right)$ to $P_{s}(v, \cdot)$.

We denote by $u^{n}$ and $u$ the solutions of (3.2) with initial data $v^{n}$ and $v$ respectively. In order to show this convergence we prove that we have

$$
\sup _{t \in[0,1]}\left(\left\|u_{t}^{n}-u_{t}\right\|_{H^{1}}+\left\|u_{s_{n}}-u_{s}\right\|_{H^{1}}\right) \rightarrow 0, \mathbb{P} \text {-a.s. }
$$

The convergence $\left\|u_{s_{n}}-u_{s}\right\|_{H^{1}} \rightarrow 0$ is a direct consequence of $u \in C\left([0,1], H^{1}\right)$, $\mathbb{P}$-a.s. It is shown in [DD] that

$$
\int_{0}^{\cdot} S^{\lambda}(\cdot-r) \Phi d W_{r} \in C\left([0,1] ; H^{1}\left(\mathbb{R}^{d}\right)\right) \cap L^{4(\sigma+1) / \sigma d}\left(0,1, W^{1,2 \sigma+2}\left(\mathbb{R}^{d}\right)\right) \quad \mathbb{P} \text {-a.s. }
$$

Thus, applying [DD, Proposition 3.5], we also have $\mathbb{P}_{\text {-a.s. }}\left|u^{n}-u\right|_{C\left([0,1] ; H^{1}\left(\mathbb{R}^{d}\right)\right)} \rightarrow 0$ as $n \rightarrow \infty$.

We now show that (5.22) implies the convergence $P_{s_{n}}\left(v^{n}, \cdot\right) \rightarrow P_{s}(v, \cdot)$. We pick $\xi: H^{1}\left(\mathbb{R}^{d}\right) \rightarrow \mathbb{R}$ uniformly continuous and bounded. Then

$$
\begin{aligned}
\left|P_{s} \xi(v)-P_{s_{n}} \xi\left(v^{n}\right)\right| & \leq \mathbb{E}\left[\left|\xi\left(u_{s}\right)-\xi\left(u_{s_{n}}^{n}\right)\right|\right] \\
& \leq \mathbb{E}\left[\left|\xi\left(u_{s}\right)-\xi\left(u_{s_{n}}\right)\right|\right]+\mathbb{E}\left[\left|\xi\left(u_{s_{n}}\right)-\xi\left(u_{s_{n}}^{n}\right)\right|\right] .
\end{aligned}
$$


Note that (5.22) and the uniform continuity of $\xi$ imply that $\mathbb{P}$-a.s. $\left|\xi\left(u_{s}\right)-\xi\left(u_{s_{n}}\right)\right|+\left|\xi\left(u_{s_{n}}\right)-\xi\left(u_{s_{n}}^{n}\right)\right| \rightarrow 0$ as $n \rightarrow \infty$. By the dominated convergence theorem, we obtain $\left|P_{s} \xi(v)-P_{s_{n}} \xi\left(v^{n}\right)\right| \rightarrow 0$ as $n \rightarrow \infty$.

\section{Compactness of the set of invariant measures}

In this section, we establish the existence of an ergodic measure.

Theorem 6.1. Under Assumptions 3.1, the set of $H^{1}\left(\mathbb{R}^{d}\right)$-valued invariant measures is a convex and compact subset of the space of probability measures on $H^{1}\left(\mathbb{R}^{d}\right)$.

Proof. Note that the convexity is trivial, so we only need to show compactness. Let $\mu$ be such a measure and $(u(t))$ the solution of (3.2) having distribution $\mu$ at all time. For simplicity of notation, we denote $M_{s}=M(u(s))$ and $H_{s}=H(u(s))$. Our first objective is to prove the integrability of these semimartingales.

We fix $R_{0}, R>0$ and define $\tau_{R}:=\inf \left\{s \geq 0: M_{s} \geq R\right\}$. We apply (3.5) on the event $\left\{M_{0} \leq R_{0}\right\}$ and obtain

$$
\begin{aligned}
M_{t \wedge \tau_{R}}= & M_{0} e^{-2 \lambda t \wedge \tau_{R}}+\|\Phi\|_{H S\left(L^{2} ; L^{2}\right)}^{2} \int_{0}^{t \wedge \tau_{R}} e^{-2 \lambda\left(t \wedge \tau_{R}-s\right)} d s \\
& +2 \int_{0}^{t \wedge \tau_{R}} e^{-2 \lambda\left(t \wedge \tau_{R}-s\right)} \operatorname{Re}\left(u(s), \Phi e_{i}\right) d B_{s}^{i} .
\end{aligned}
$$

Note that by the localization the expectation of the stochastic integral vanishes. Therefore,

$$
\mathbb{E}\left[M_{t \wedge \tau_{R}} \mathbf{1}_{\left\{M_{0} \leq R_{0}\right\}}\right]=\mathbb{E}\left[M_{0} \mathbf{1}_{\left\{M_{0} \leq R_{0}\right\}} e^{-2 \lambda t \wedge \tau_{R}}\right]+\|\Phi\|_{H S\left(L^{2} ; L^{2}\right)}^{2} \mathbb{E}\left[\int_{0}^{t \wedge \tau_{R}} e^{-2 \lambda\left(t \wedge \tau_{R}-s\right)} \mathbf{1}_{\left\{M_{0} \leq R_{0}\right\}} d s\right] .
$$

For fixed $R_{0}$, the integrands on the right hand side are uniformly bounded and the integrand on the left hand side is non-negative. We apply the dominated convergence theorem for the right side and Fatou's lemma for the left to obtain that

$$
\mathbb{E}\left[M_{t} \mathbf{1}_{\left\{M_{0} \leq R_{0}\right\}}\right] \leq \mathbb{E}\left[M_{0} \mathbf{1}_{\left\{M_{0} \leq R_{0}\right\}}\right] e^{-2 \lambda t}+\frac{\|\Phi\|_{H S\left(L^{2} ; L^{2}\right)}^{2}}{2 \lambda} .
$$

Therefore, we can choose $t_{R_{0}}>0$ such that for all $R_{0}>0$, we have

$$
\mathbb{E}\left[M_{t_{R_{0}}} \mathbf{1}_{\left\{M_{0} \leq R_{0}\right\}}\right] \leq \frac{\|\Phi\|_{H S\left(L^{2} ; L^{2}\right)}^{2}}{\lambda} .
$$

Noting also that the distribution of $M_{t_{R_{0}}}$ is $\mu$ we obtain there exists $f_{R_{0}}(v) \rightarrow 1 \mu$-a.s. as $R_{0} \rightarrow \infty$ and

$$
\mathbb{E}\left[M_{t_{R_{0}}} \mathbf{1}_{\left\{M_{0} \leq R_{0}\right\}}\right]=\int\|v\|_{L^{2}}^{2} f_{R_{0}}(v) \mu(d v) \leq \frac{\|\Phi\|_{H S\left(L^{2} ; L^{2}\right)}^{2}}{\lambda} .
$$

Taking the limit $R_{0} \rightarrow \infty$, we obtain

$$
\int\|v\|_{L^{2}}^{2} \mu(d v) \leq \frac{\|\Phi\|_{H S\left(L^{2} ; L^{2}\right)}^{2}}{\lambda}
$$


Similarly to the proof of Lemma 5.1, we apply Ito's lemma to $M^{k+1}(u(t))$, localize with stopping times and prove that there exists $C_{k}(\Phi, \lambda)$ which may a priori depend on $\mu$ such that

$$
\int\|v\|_{L^{2}}^{2 k} \mu(d v) \leq C_{k}(\Phi, \lambda)<\infty, \quad k=1 \ldots
$$

We also apply the same procedure to $H_{t}$ to obtain that there exists $\widetilde{C}_{k}(\Phi, \lambda)$ that may again depend on $\mu$ such that

$$
\int\|v\|_{H^{1}}^{2 k} \mu(d v) \leq \widetilde{C}_{k}(\Phi, \lambda)<\infty .
$$

Given this integrability, we return to (3.5) and(3.6) to prove that $C_{k}(\Phi, \lambda)$ and $\widetilde{C}_{k}(\Phi, \lambda)$ can be taken independent of $\mu$. Since $\mu$ is an invariant measure, we get $d \mathbb{E}\left[M_{t}\right]=d \mathbb{E}\left[H_{t}\right]=0$ and

$$
\mathbb{E}\left[M_{t}\right]=\frac{\|\Phi\|_{H S\left(L^{2} ; L^{2}\right)}^{2}}{2 \lambda} .
$$

Using the same invariance we obtain

$$
\begin{aligned}
2 \lambda \mathbb{E}\left[M_{t}^{k+1}\right] & =\|\Phi\|_{H S\left(L^{2}, L^{2}\right)}^{2} \mathbb{E}\left[M_{t}^{k}\right]+\frac{k}{2} \mathbb{E}\left[M_{t}^{k-1} \sum_{i} \operatorname{Re}\left(u(t), \Phi e_{i}\right)^{2}\right] \\
& \leq\left(\|\Phi\|_{H S\left(L^{2}, L^{2}\right)}^{2}+\frac{k}{2}\right) \mathbb{E}\left[M_{t}^{k}\right],
\end{aligned}
$$

which shows by induction that $C_{k}(\Phi, \lambda)$ may be taken independent of $\mu$. Applying the same procedure to the equation (3.6), we obtain that $\widetilde{C}_{k}(\Phi, \lambda)$ can be taken independent of $\mu$.

We now prove the sequential compactness of the set of $H^{1}\left(\mathbb{R}^{d}\right)$-valued invariant measures. Let $\mu^{n}$ be a sequence of such invariant measures of the equation (3.2). Without loss of generality, we assume that the $\sigma$-algebra $\mathbb{F}_{0}$ is rich enough so that there exists a family of $\mathbb{F}_{0}$-measurable random variables $u_{0}^{n}$ with distribution $\mu^{n}$. The uniform bounds we have proven gives us

$$
\sup _{n} \int\|v\|_{L^{2}}^{2 k} \mu^{n}(d v) \leq C_{k}(\Phi, \lambda)
$$

and

which a fortiori imply

$$
\sup _{n} \int\|v\|_{H^{1}}^{2} \mu^{n}(d v) \leq \widetilde{C}_{k}(\Phi, \lambda)
$$

$$
\mathbb{E}\left[\left\|u_{0}^{n}\right\|_{H^{1}}^{4 \vee\lceil 4 d \sigma\rceil}+\left\|u_{0}^{n}\right\|_{L^{2}}^{\lceil 4 \sigma(2-d)+8\rceil}\right] \leq R .
$$

Therefore, Lemma 3.5 and the fact that $\mu^{n}$ is an invariant measure show that the family

$$
\left\{\left(\mu^{n} P_{t_{n}}\right)(\cdot): n \in \mathbb{N}\right\}=\left\{\mu^{n}: n \in \mathbb{N}\right\}
$$

is tight. Noting that the set of invariant measures is closed, we obtain the required compactness.

Corollary 6.2. Under Assumptions [3.1, there exists an ergodic invariant measure.

Proof. By the Krein-Milman theorem, the compactness of the set of invariant measures implies that there exists at least one invariant measure that is an extremal point of this set. Proposition 3.2.7 of [DZ] then implies that such a measure is ergodic. 


\section{Acknowledgments}

I.K. was supported in part by the NSF grant DMS-1311943, while M.Z. was supported in part by the NSF grant DMS-1109562.

\section{References}

[B] J.M. Ball, Continuity properties and global attractors of generalized semiflows and the NavierStokes equations, Mechanics: from theory to computation, Springer, New York, 2000, pp. 447474 .

[Bi] P. Billingsley, Convergence of probability measures, second ed., Wiley Series in Probability and Statistics: Probability and Statistics, John Wiley \& Sons, Inc., New York, 1999, A WileyInterscience Publication.

[CGV] P. Constantin, N. Glatt-Holtz, and V. Vicol, Unique ergodicity for fractionally dissipated, stochastically forced 2D Euler equations, Comm. Math. Phys. 330 (2014), no. 2, 819-857.

[DZ] G. Da Prato and J. Zabczyk, Ergodicity for infinite-dimensional systems, London Mathematical Society Lecture Note Series, vol. 229, Cambridge University Press, Cambridge, 1996.

[DD] A. de Bouard and A. Debussche. The stochastic nonlinear Schrödinger equation in $H^{1}$, Stochastic Analysis and Applications, Vol. 21, Iss. 1, (2003).

[DO] A. Debussche and C. Odasso, Ergodicity for a weakly damped stochastic non-linear Schrödinger equation, J. Evol. Equ. 5 (2005), no. 3, 317-356.

[DV] A. Debussche and J. Vovelle, Scalar conservation laws with stochastic forcing, J. Funct. Anal. 259 (2010), no. 4, 1014-1042.

[D] E. Dettweiler, Representation of Banach space valued martingales as stochastic integrals, Probability in Banach spaces, 7 (Oberwolfach, 1988), Progr. Probab., vol. 21, Birkhäuser Boston, Boston, MA, 1990, pp. 43-62.

[EKZ] I. Ekren, I. Kukavica, and M. Ziane, Existence of invariant measures for the stochastic damped KdV equation (2015) arXiv:1512.02686.

[F1] F. Flandoli, Dissipativity and invariant measures for stochastic Navier-Stokes equations, NoDEA Nonlinear Differential Equations Appl. 1 (1994), no. 4, 403-423.

[F2] F. Flandoli, An introduction to 3D stochastic fluid dynamics, SPDE in hydrodynamic: recent progress and prospects, Lecture Notes in Math., vol. 1942, Springer, Berlin, 2008, pp. 51-150.

[GKVZ] N. Glatt-Holtz, I. Kukavica, V. Vicol, and M. Ziane, Existence and regularity of invariant measures for the three dimensional stochastic primitive equations J. Math. Phys. 55 (2014), $34 \mathrm{pp}$. 
[GMR] N. Glatt-Holtz, J. Mattingly, and G. Richards, On unique ergodicity in nonlinear stochastic partial differential equations, arXiv: 1512.04126v1.

[G1] O. Goubet, Regularity of the attractor for a weakly damped nonlinear Schrödinger equation, Appl. Anal. 60 (1996), no. 1-2, 99-119.

[G2] O. Goubet, Regularity of the attractor for a weakly damped nonlinear Schrödinger equation in $\mathbf{R}^{2}$, Adv. Differential Equations 3 (1998), no. 3, 337-360.

[G3] O. Goubet, Asymptotic smoothing effect for weakly damped forced Korteweg-de Vries equations, Discrete Contin. Dynam. Systems 6 (2000), no. 3, 625-644.

[GM] O. Goubet and I. Moise, Attractor for dissipative Zakharov system, Nonlinear Anal. 31 (1998), no. $7,823-847$.

[GK] O. Goubet and W. Kechiche, Uniform attractor for non-autonomous nonlinear Schrödinger equation, Commun. Pure Appl. Anal. 10 (2011), no. 2, 639-651.

[GL] O. Goubet and L. Legry, Existence of a finite-dimensional global attractor for a damped parametric nonlinear Schrödinger equation, Adv. Differential Equations 17 (2012), no. 9-10, 859877 .

[GR] O. Goubet and R.M.S. Rosa, Asymptotic smoothing and the global attractor of a weakly damped $K d V$ equation on the real line, J. Differential Equations 185 (2002), no. 1, 25-53.

[HM] M. Hairer and J.C. Mattingly, Ergodicity of the 2D Navier-Stokes equations with degenerate stochastic forcing, Ann. of Math. (2) 164 (2006), no. 3, 993-1032.

[KS] S. Kuksin and A. Shirikyan, Ergodicity for the randomly forced $2 D$ Navier-Stokes equations, Math. Phys. Anal. Geom. 4 (2001), no. 2, 147-195.

[MR] C.M. Mora and R. Rebolledo, Basic properties of nonlinear stochastic Schrödinger equations driven by Brownian motions, Ann. Appl. Probab. 18 (2008), no. 2, 591-619.

[MiR1] R. Mikulevicius and B.L. Rozovskii, Martingale problems for stochastic PDE's, Stochastic partial differential equations: six perspectives, Math. Surveys Monogr., vol. 64, Amer. Math. Soc., Providence, RI, 1999, pp. 243-325.

[MiR2] R. Mikulevicius and B.L. Rozovskii, Global L L $_{2}$-solutions of stochastic Navier-Stokes equations, Ann. Probab. 33 (2005), no. 1, 137-176.

[P] J. Printems, The stochastic Korteweg-de Vries equation in $L^{2}(\mathbf{R})$, J. Differential Equations 153 (1999), no. 2, 338-373.

[Pr] Y. Prokhorov, Convergence of random processes and limit theorems in probability theory, Theory of Prob. and Appl. 2 (1956), no. 2,157-214. 
[R] R. Rosa, The global attractor of a weakly damped, forced Korteweg-de Vries equation in $H^{1}(\mathbb{R})$, Mat. Contemp. 19 (2000), 129-152, VI Workshop on Partial Differential Equations, Part II (Rio de Janeiro, 1999).

[T] R. Temam, Sur un problème non linéaire, J. Math. Pures Appl. (9) 48 (1969), 159-172. 